\title{
JOINT OFFSHORE WIND FIELD MONITORING WITH SPACEBORNE SAR AND PLATFORM-BASED DOPPLER LIDAR MEASUREMENTS
}

\author{
S. Jacobsen ${ }^{\text {a, }}$, S. Lehner ${ }^{\text {a }}$, J. Hieronimus ${ }^{\text {b }}$, J. Schneemann ${ }^{\text {b }}$, M. Kühn ${ }^{\text {b }}$ \\ ${ }^{\text {a }}$ German Aerospace Center (DLR), Remote Sensing Technology Institute, Bremen, Germany - Sven.Jacobsen@dlr.de \\ ${ }^{\mathrm{b}}$ ForWind - Center for Wind Energy Research, Oldenburg, Germany - J.Schneemann@uni-oldenburg.de
}

\author{
Commission VI, WG VI/4
}

KEY WORDS: SAR, Wind, Energy, Turbine, Wake, LiDAR

\begin{abstract}
:
The increasing demand for renewable energy resources has promoted the construction of offshore wind farms e.g. in the North Sea. While the wind farm layout consists of an array of large turbines, the interrelation of wind turbine wakes with the remaining array is of substantial interest. The downstream spatial evolution of turbulent wind turbine wakes is very complex and depends on manifold parameters such as wind speed, wind direction and ambient atmospheric stability conditions.

To complement and validate existing numerical models, corresponding observations are needed. While in-situ measurements with e.g. anemometers provide a time-series at the given location, the merits of ground-based and space- or airborne remote sensing techniques are indisputable in terms of spatial coverage. Active microwave devices, such as Scatterometer and Synthetic Aperture Radar (SAR), have proven their capabilities of providing sea surface wind measurements and particularly SAR images reveal wind variations at a high spatial resolution while retaining the large coverage area. Platform-based Doppler LiDAR can resolve wind fields with a high spatial coverage and repetition rates of seconds to minutes. In order to study the capabilities of both methods for the investigation of small scale wind field structures, we present a direct comparison of observations obtained by high resolution TerraSAR-X (TS-X) X-band SAR data and platform-based LiDAR devices at the North Sea wind farm alpha ventus. We furthermore compare the results with meteorological data from the COSMO-DE model run by the German Weather Service DWD. Our study indicates that the overall agreement between SAR and LiDAR wind fields is good and that under appropriate conditions small scale wind field variations compare significantly well.
\end{abstract}

\section{INTRODUCTION}

\subsection{Introduction}

The coastal zones of Germany represent an essential constituent in the economic and social development, such as the extensive exploitation of offshore wind energy. In the planning and construction process of new wind farms, high resolution meteomarine parameters can contribute in manifold ways like providing margins for the expected energy potential on the basis of long-term statistical analysis of wind fields (Sempreviva, 2008). Monitoring of existing wind farms also provides extremely useful information. Investigation of the turbulent wake properties can help to optimize future designs of turbine array geometries and maximize energy output. Moreover, despite a number of existing offshore wind farms, the environmental impact of the facilities on the coastal zones has not yet been extensively investigated. Turbine wake observations can also contribute in the assessment of possible impact areas.

Over the past four decades, spaceborne remote sensing has developed to a mature technology. It is now playing an important role in Earth Observation (EO) due to its capabilities of global coverage (including remote and poorly accessible regions) and possibilities of long-term observations. Particularly microwave sensors have become increasingly popular as the signals can penetrate clouds and are thus widely independent of weather conditions. Especially sea surface wind speed is one parameter commonly derived from microwave sensors. While radiometers and scatterometers yield only coarse resolution data, synthetic aperture radar (SAR) has proven to measure local wind related sea surface roughness with both, high resolution and wide coverage (Lehner, 1998; Horstmann, 2004).
On the other hand, earth-bound remote sensing techniques have evolved significantly. Especially Doppler LiDAR has become an important tool for offshore wind energy related research in the last years (Peña, 2009). Ground-based LiDAR windscanners enable to measure vertical wind profiles or horizontal planar scans of the radial wind speed within a range of several kilometres.

SAR and LiDAR have been applied to offshore wind farm monitoring (Trabucchi 2013; Li, 2013). While both methods have been successfully validated with meteorological models and in-situ measurements, the work presented in this paper is to our knowledge the first direct comparison of platform based LiDAR measurements and 2D sea surface wind fields derived from spaceborne SAR.

Both methods are evaluated with a special focus on the detection of small scale variations in wind fields and the analysis is supported by a comparison with data from the numerical meteorologial model COSMO-DE run by DWD.

\section{METHODS}

\subsection{SAR Observations by TerraSAR-X}

The SAR satellite TerraSAR-X sends radar pulses at a certain incidence angle towards the ocean surface. The backscatter to the sensor from the rough sea surface is explained by Bragg scattering caused by small capillary waves that are in resonance with the impinging radiation. As the small scale sea surface roughness is directly related to surface wind conditions, the radar return measured in normalized radar cross-section (NRCS) can be used to infer wind fields from radar images. As the relationship is dependent on many factors, wind field estimation is traditionally performed with geophysical model 


\begin{tabular}{|c|c|c|c|c|}
\hline Date & 16.01. & 17.01. & 19.01. & 22.01. \\
\hline Modus & Spot Light & Spot Light & Spot Light & Strip Map \\
\hline Resolution & $60 \mathrm{~m}$ & $60 \mathrm{~m}$ & $60 \mathrm{~m}$ & $60 \mathrm{~m}$ \\
\hline Time & $05: 59$ & $05: 42$ & $17: 27$ & $05: 51$ \\
\hline Scene Size & $10 \times 12 \mathrm{~km}$ & $10 \times 12 \mathrm{~km}$ & $10 \times 12 \mathrm{~km}$ & $28 \times 31 \mathrm{~km}$ \\
\hline $\begin{array}{c}\text { Incidence } \\
\text { angle }\end{array}$ & $28^{\circ}$ & $49^{\circ}$ & $51^{\circ}$ & $38^{\circ}-40^{\circ}$ \\
\hline
\end{tabular}

Table 1: TS-X acquisition parameters on measurement dates

functions (GMFs) (Stoffelen 1997; Hersbach 2004; Hersbach 2007a). The GMFs relate the NRCS to a combination of wind speed and wind direction. As both parameters are unknown, it is common practice to either extract the wind direction from distinct SAR image features like wind streaks if present, or to use information derived from meteorological model data.

The X-band SAR satellite TerraSAR-X (TS-X) was launched in June 2007 and its twin TanDEM-X (TD-X) in June 2010. TS-X and TD-X operate from $514 \mathrm{~km}$ height at sun-synchronous orbits, the ground speed is $7 \mathrm{~km} / \mathrm{s}$ (15 orbits per day). Both satellites are orbiting in a close formation with typical distances between satellites of $250 \mathrm{~m}$ to $500 \mathrm{~m}$. They operate with a wavelength of $31 \mathrm{~mm}$ and frequency of $9.6 \mathrm{GHz}$. The repeatcycle is 11 days, but the same region can be imaged with different incidence angles after three days dependent on scene latitude. Typical incidence angles range between $20^{\circ}$ and $55^{\circ}$. The coverage and resolution depends on the satellite mode: StripMap covers $30 \mathrm{~km}$ by $50 \mathrm{~km}$ with a resolution of about $3 \mathrm{~m}$, Spotlight covers $10 \mathrm{~km}$ by $10 \mathrm{~km}$ with resolution of about $1 \mathrm{~m}$ (Breit 2010).

An adaption of the well-established CMOD5 algorithm (Hersbach, 2007a) for X-band was recently developed for TS$\mathrm{X}$ : XMOD2 (Li, 2014). With this next-generation algorithm, major progress in the wind analysis quality was achieved by retuning the coefficients of the GMFs for X-Band, using colocated buoy measurements. The output is the local wind field at $10 \mathrm{~m}$ height. XMOD2 has been successfully validated against independent buoy data and meteorological models operated by the German weather service DWD. The mean accuracy of the XMOD2 results was identified to be better than $1.46 \mathrm{~m} / \mathrm{s}$ in terms of RMSE (Li, 2014). Hence, TS-X images, interpreted with the XMOD2 algorithm provide a unique opportunity of measuring sea surface wind fields with high resolution and accuracy.

Wind fields in $10 \mathrm{~m}$ height with a spatial resolution of $60 \mathrm{~m}$ have been calculated at the offshore wind farm alpha ventus. Satellite acquisitions were taken around 6:00 (UTC) on three occasions and at 17:27 (UTC) on one occasion. The TS-X acquisition parameters on the different dates are summarized in Table 1.

\subsection{COSMO-DE Reanalysis Data}

The COSMO-DE mesoscale weather model of the German Weather Service (DWD, www.dwd.de), delivers instantaneous wind speed values and atmospheric information on a horizontal grid with $2.8 \mathrm{~km}$ resolution and 1 hour time step. COSMO-DE periodically updates the simulation by assimilation of real measured data into the simulation every 3 hours, which makes a good estimate of the atmospheric state possible. However, deviations between measured and simulated reanalysis data have to be considered, since the simulation gives a best fit of the weather situation over a larger area but not at a specific point. (Baldauf 2011)

From the COSMO-DE model analysis data about the vertical wind profile and the surface roughness parameter $z_{0}$ is used in this contribution. The vertical wind profile given by the

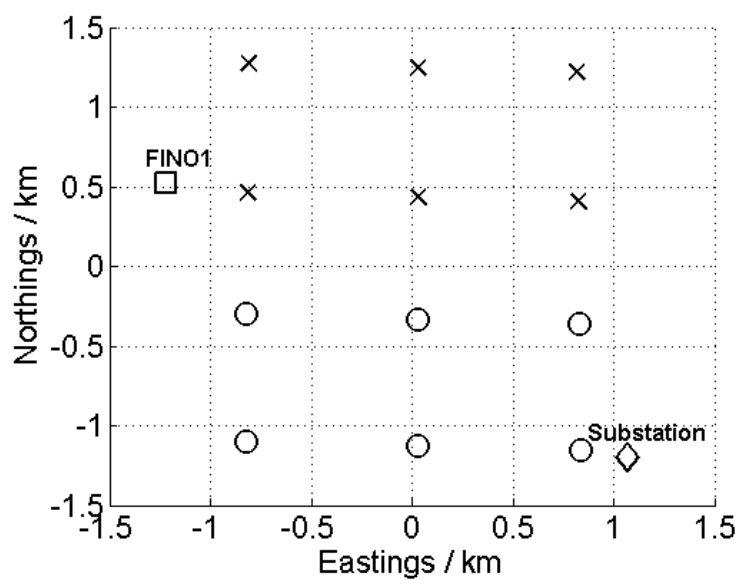

Figure 1. Layout of the offshore wind farm alpha ventus. Crosses indicate positions of Senvion $5 M$ turbines with a diameter of $126 \mathrm{~m}$ (two northerly rows). Circles indicate positions of the AREVA Wind M5000 turbines with a diameter of $116 \mathrm{~m}$ (two southerly rows). One LiDAR was operated on the substation $(\diamond)$ and two on the research platform Fino1 ( $\square$ ).

COSMO parameters $\mathrm{U}$ and $\mathrm{V}$ is used for the height levels at $10 \mathrm{~m}, 35 \mathrm{~m}, 73 \mathrm{~m}, 122 \mathrm{~m}$ and $184 \mathrm{~m}$.

\subsection{LiDAR Measurements}

Doppler LiDAR devices send out laser pulses to the atmosphere and receive the signal backscattered by aerosol particles moving with the airflow. A Doppler shift in the signal being proportional to the line-of-sight component of the aerosol's speed is detected and analysed by the LiDAR. (Werner 2005). The long range scanning Doppler Lidar Leosphere Windcube200S emits infrared Laser pulses with a wave length of $1.54 \mu \mathrm{m}$. The flexible settings in terms of averaging time, selectable pulse length and scanning speed allow radial wind speed measurements up to a distance of $6.5 \mathrm{~km}$. More than 200 measurements points in different distances along the laser beam can be processed.

For the experiment three Windcube200S LiDAR systems have been installed and operated in the offshore wind farm alpha ventus in the German North Sea from July 2013 to March 2014. Two LiDAR were installed on the research platform and meteorological mast FINO1at 20 and $22 \mathrm{~m}$ above sea level and one LiDAR was located on the substation of the wind farm at $25 \mathrm{~m}$ above sea level. The layout of the wind farm is shown in Figure 1.

The trajectory of the LiDAR was set to the so called PPI (plan position indicator) mode, measuring wind speeds in the horizontal plane with changing azimuth and a small fixed elevation angle, leading to varying measurement heights between $25 \mathrm{~m}$ and $80 \mathrm{~m}$. The scanning speed was set to $1 \% \mathrm{~s}$ with an averaging time of $1 \mathrm{~s}$. The whole azimuth range was scanned by all systems except for some sectors with blocked sight. In total 198 equally spaced range gates from $100 \mathrm{~m}$ to $6000 \mathrm{~m}$ range have been measured by each LiDAR. In figure 2 the filtered LoS-wind speeds of one LiDAR PPI scan are plotted. For comparison a velocity field of TS-X is shown in Figure 3, illustrating the wake development behind the wind turbines measured by both systems. Only the data of the LiDAR located on the wind farm`s substation is presented in this contribution for the comparison with TS-X. 


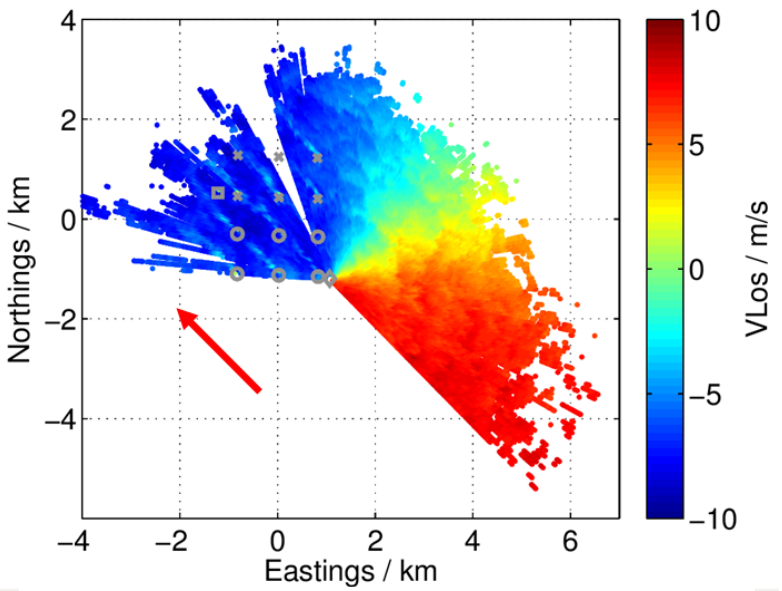

Figure 2. Line of sight velocity measured by LiDAR on sub station on January 22. 2014. The red arrow illustrated the wind direction based on the COSMO-DE model.

\subsection{Data Analysis}

A direct comparison of wind speed measurements of TS-X to data obtained from LiDAR measurements or reanalysis data is difficult, since the data is provided in different spatial resolution and at different times. Moreover, data values are given at different heights. For this reasons all data sets were interpolated to a reference grid and extrapolated to $10 \mathrm{~m}$ height assuming a logarithmic wind profile.

To compare TS-X and COSMO-DE wind fields, the TS-X data was downsampled, since the resolution of the wind field grid are $60 \mathrm{~m}$ for the TS-X and $2.8 \mathrm{~km}$ for the COSMO data, respectively. For the comparison, TS-X data within a half COSMO cell width around each grid centre from the COSMO grid was selected and averaged. Values containing the area of the the wind farm alpha ventus were not considered for comparison, for two reasons. First, the strong radar echoes from offshore structures contaminate the TS-X data and the derived windfield and secondly, the influence of alpha ventus on the wind field is not considered in COSMO-DE, yet visible in TS-X data. Both factors would cause a mismatch and hamper a reliable comparison. After downsampling and exclusion of questionable locations, the mean difference and standard deviation of the wind fields is calculated.

For the investigation of differences in the TS-X and the LiDAR wind fields a radial grid in $10 \mathrm{~m}$ height serves as reference grid. The general method for comparison is to calculate the absolute horizontal wind speed from LiDAR data and to transfer it to the reference grid by linear interpolation. Subsequently an extrapolation to the $10 \mathrm{~m}$ level is performed assuming a neutral logarithmic profile.

In a first step, LiDAR data is pre-processed by removing bad CNR (carrier to noise ratio) values and selecting a maximum time difference of $120 \mathrm{~s}$ with respect to the TS-X acquisition. Furthermore, the data is separated into two qualitatively different subsets: One is determined by locations upstream the turbine array with respect to the ambient wind direction and is thus considered to represent free stream conditions, the other subset is located downstream and likely affected by the wind farm. Additional meteorological information about the ambient wind direction and surface roughness parameter $z_{0}$ at the wind farm alpha vetnus is needed for the data analysis. The parameter was taken from the weather model COSMO-DE, since the meteorological mast FINO1 was in the wake of the wind farm in all situations considered here. For calculation of the absolute horizontal wind $v_{m}$ from the line of sight (LoS) measurements of the LiDAR, all measured LoS wind speeds $v_{\text {LOS }}$ are projected

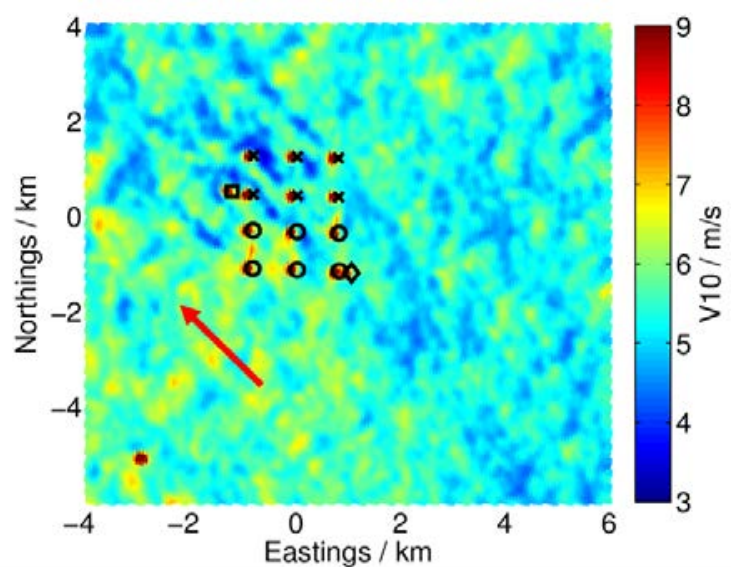

Figure 3. Seas surface wind field derived from a TerraSAR-X StripMap scene over alpha ventus, January 22, 2014. The red arrow illustrated the wind direction based on COSMO-DE.

to the mean horizontal wind direction obtained from COSMODE using

$$
v_{m}=\frac{v_{L o S}}{\cos \alpha}
$$

with $\alpha$ being the angle between the LoS direction and the wind direction. The accuracy of this method decreases significantly with $\alpha$ approaching values of 90 degrees. Consequently sectors with $|\alpha|>40^{\circ}$ are neglected in the further analysis. The calculated absolute horizontal wind speed is transferred to the planar radial reference grid with a resolution of $60 \mathrm{~m}$ in radial and $1^{\circ}$ in azimuthal direction centred at the LiDAR position. On each grid point the corresponding averaged horizontal wind speed $v_{m}$ and height level is estimated by averaging all calculated absolute wind speed data within each grid cell. All wind speeds of the reference grid are extrapolated to the height $z=10 \mathrm{~m}$ using the logarithmic wind profile for neutral conditions

$$
v(z)=v_{m} \frac{\ln \left({ }^{z} / z_{0}\right)}{\ln \left({ }^{z} / z_{0}\right)}
$$

with the height $z$, the measurement height $z_{m}$ of the LiDAR, the wind speed $v_{m}$ in measurement height and the surface roughness $z_{0}$. Offshore, the surface roughness is dependent on the swell with typical values ranging from $z_{0}=1 \cdot 10^{-5} \mathrm{~m}$ to $z_{0}=1 \cdot 10^{-3} \mathrm{~m}$ (Emeis 2012).

The model used in Equation 2 is valid for a neutral stratification of the atmosphere, which introduces a further error in the case of other stratifications. However, as dependable data to evaluate the stability of the atmosphere was not available, the neutral logarithmic profile is applied as a first approach.

For the comparison the difference between the extrapolated LiDAR wind field and the TS-X wind field is calculated for free stream conditions and averaged in the horizontal plane, resulting in a value for the mean and standard deviation of the difference between both wind fields. Furthermore, LiDAR and radar satellite are compared with COSMO-DE data. As most of the LiDAR data is located in one grid cell of COMSO-DE, for convenience only the $10 \mathrm{~m}$ wind speed value at the midpoint of the wind farm array is used instead of a spatial comparison as described earlier.

In wake conditions, the logarithmic wind profile used to transfer the wind speeds of the LiDAR to the height of the TS-X values does not hold valid and thus a comparison of wind speeds is disputable. Due to this reason in the analysis just the wake position are investigated by interpolation of LiDAR and TS-X 
data to a Cartesian grid covering the wake area downstream of the turbine. The maximum deficit of the wake is searched in several distances downstream fitting a Gaussian function to lines perpendicular to the wake.

\subsection{Comparison of measurement uncertainties}

The two remote sensing methods compared in this article exhibit fundamental differences. While the space borne SAR satellite uses radar to investigate the roughness of the sea surface and relates this to the sea surface wind speed a ground based Doppler LiDAR uses laser light to measure radial speeds of aerosols in the atmosphere from which the absolute wind speed is derived. Depending on the area coverage a scan of the LiDAR takes up to some minutes while TS-X takes an image in about $1 \mathrm{~s}$. Measurements of both systems comprise different sources of errors.

Radar satellites follow the approach to indirectly measure the wind speed by careful observation of wind wave interaction
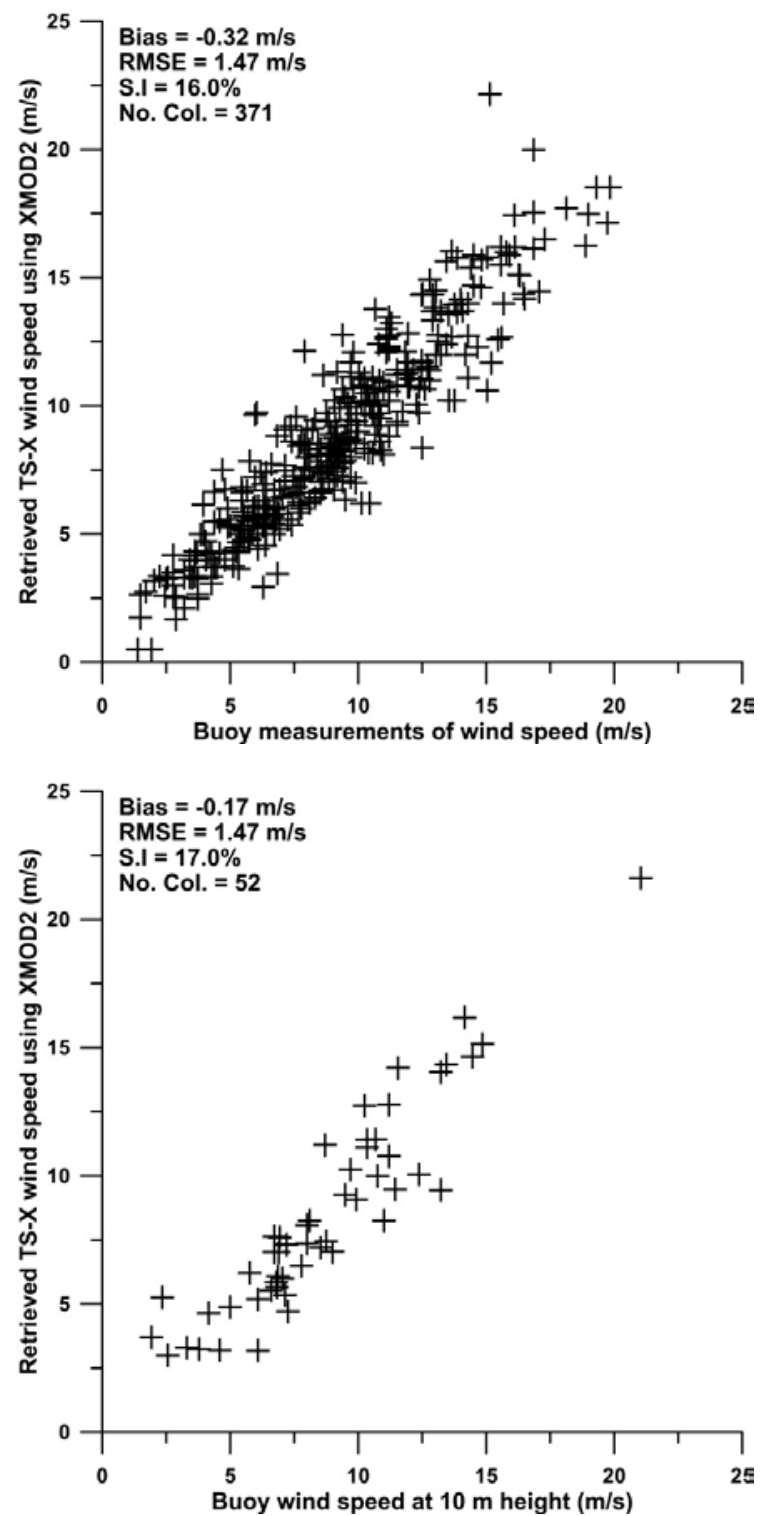

Figure 4: Comparison of the retrieved TS-X/TD-X sea surface wind speed using XMOD2 against in situ buoy measurements (Top: Tuning data set, Bottom: Validation data set) - after Li, 2014. affecting the backscatter intensity of the ocean surface. However, this parameter is not only influenced by wind, but is altered by surface films (e.g. oil), strong ocean currents, upwelling and the like. In particular rain can have an impact on the received backscatter in two different ways. Either, the direct effect of rain drops on the ocean surface increase the roughness and backscatter, or the indirect effect of big rain drops in clouds that already scatter the impinging radar pulse, resulting in apparently lower backscatter from the sea surface as less energy arrives. A further uncertainty lies within the method itself. The GMFs in the XMOD2 algorithm and similar C-band predecessors empirically connect sea surface roughness to measured wind speeds at $10 \mathrm{~m}$ height. As the vertical wind profile varies with stratification conditions, the empirical approach yields a good wind field for the average stratification present in the tuning data, but these do not necessarily match the conditions at a particular scene under investigation. Moreover, the extrapolation of in-situ buoy wind measurements to $10 \mathrm{~m}$ for algorithm tuning and validation apply assumptions about the atmospheric stability and hence the GMFs inherit possible errors in this assumption for certain occasions. However, the scatterplots in Figure 4 for tuning and validation (taken from $\mathrm{Li}$ and Lehner 2014) illustrate the good statistical agreement with an RMSE of $1.46 \mathrm{~m} / \mathrm{s}$ (for the validation data) but also exhibit a larger offset for single observations. Another uncertainty in the wind field generation from SAR data is the wind direction fed into the algorithm. An incorrect wind direction leads to differences in the wind speed output. However, the effect of this is minor for deviations of a few degrees. An example of the XMOD2 results for an incidence angle of $35^{\circ}$ is shown in Figure 5.

Doppler Lidar Systems measure the radial wind speed directly by illuminating aerosols. For this measurement principle it is assumed that lightweight aerosol particles move in the same direction and speed as the wind. The overall measurement error for the projection considered in this contribution is found by

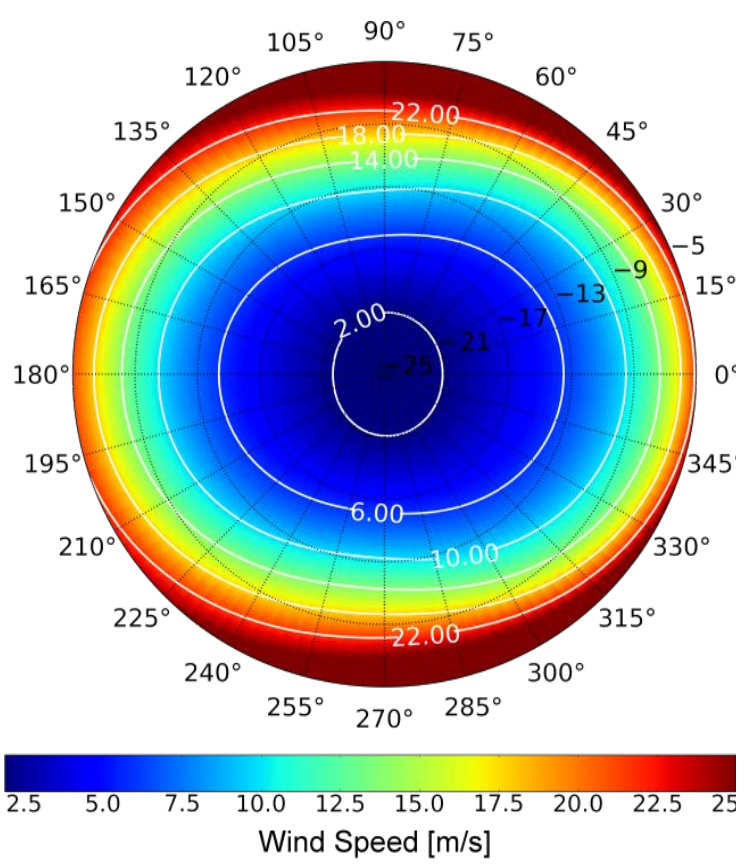

Figure 5. Wind speed derived from XMOD2 for an incidence angle of $35^{\circ}$. Radial axis/ black circles: NRCS in $\mathrm{dB}$. Angle: Wind direction relative to antenna look direction at $0^{\circ}$. Color code: Wind speed in $\mathrm{m} / \mathrm{s}$. 
error propagation of Equation 1.

$$
\Delta v_{m}=\left|\frac{1}{\cos (\alpha)}\right| \Delta v_{L O S}+\left|\frac{v_{L O S} \sin (\alpha)}{\cos ^{2}(\alpha)}\right| \Delta \alpha
$$

The measurement error is composed of the line of sight error $\Delta v_{L O S}$ and the angle error $\Delta \alpha$, with $\alpha$ being the difference between the angle $\vartheta$ of the real wind speed and the azimuth angle $a z$ of the line of sight velocity. The line of sight error is a fixed attribute of the LiDAR system and stems from inaccuracies of the electronics, laser resonator and detection process (Frehlich, 2001). For the Windcube $200 S$ the maximum error of $v_{L O S}$ is stated to be $0.5 \mathrm{~m} / \mathrm{s}$ by the manufacturer.

In addition to the $v_{L O S}$ measurement error for fixed azimuth or elevation angles, further measurement errors are introduced by the inaccurate determination of the scanning angle (Stawiarsky, 2012) and the wind direction needed for the projection of the line of sight velocities. These errors are summarized in $\Delta \alpha$, with $\Delta \alpha=\Delta a z-\Delta \vartheta$. The pointing accuracy $\Delta a z$ of the Windcube $200 S$ is stated to be $0.1^{\circ}$ by the manufacturer. The wind direction error $\Delta \vartheta$ results from inaccuracies from the COSMODE model. It has to be mentioned that dependable data on the wind direction error of COSMO-DE was not available but is assumed to be of the same order of direction errors of wind vanes with an value of $\Delta \vartheta=4^{\circ}$, as the simulation is based on real measurement data.

Regarding the COSMO-DE model the error cannot be assessed easily, since the simulations are based on multiple numerical models each based on individual assumptions. Recent research has been carried out in the work of Ohsawa et. al 2013 on the validation of the simulated wind speed data from COSMO-DE with in-situ measurements from the FINO1 meteorological mast. The wind speeds between COSMO-DE and FINO1 were found to be well correlated with a linear correlation coefficient of 0.95 and a RMSE of $1.36 \mathrm{~m} / \mathrm{s}$.

It can be assumed that the largest error is attributed to the height extrapolation of LiDAR data caused by non-neutral stratifications of the vertical profile. From the sea and air temperature data from the COSMO-DE model the stability can be roughly evaluated as instable on the considered times, with strongest instability occurring on the 22.1. To estimate a maximum deviation between an unstable and neutral profile a constant wind speed with height equivalent to typical conditions during maximum instability (well mixed boundary layer), is

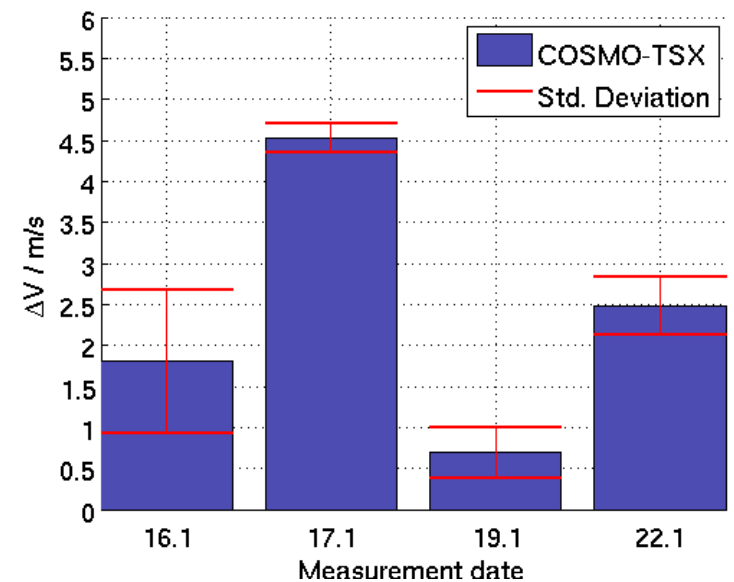

Figure 6. Comparison of $10 \mathrm{~m}$ wind fields from COSMODE and TS-X. Mean difference (height of column) and standard deviation of the difference (error bars) are shown. compared to a neutral wind speed profile. The maximum deviation between neutral and instable profile at $10 \mathrm{~m}$ height is estimated to be $16 \%$ of the corresponding non extrapolated wind speed measured by the LiDAR. It is worth to mention that the present vertical profiles were not fully instable and the maximum error is not likely to be reached. Regarding possible stable atmospheric cases higher deviations have to be taken into account, since the wind speed increase over typical rotor disk extensions can reach 150\%.(Emeis 2012).

\section{RESULTS AND DISCUSSION}

\subsection{Comparison COSMO-DE andTSX}

Figure 6 shows the comparison between the $10 \mathrm{~m}$ wind fields of COSMO-DE and TS-X for the different measurement dates. A minimal difference of the wind speed is found on the 19.01. On this date COSMO-DE wind speed is in average $0.7 \mathrm{~m} / \mathrm{s}$ higher than TS-X-based values; the standard deviation is $0.3 \mathrm{~m} / \mathrm{s}$. The maximum difference is found on the 17.01 . with a value of $4.5 \mathrm{~m} / \mathrm{s}$ and a standard deviation of $0.17 \mathrm{~m} / \mathrm{s}$. On the 16.01 . and the 22.01. mean differences of $1.8 \mathrm{~m} / \mathrm{s}$ and $2.5 \mathrm{~m} / \mathrm{s}$ are observed with standard deviations of $0.87 \mathrm{~m} / \mathrm{s}$ and $0.36 \mathrm{~m} / \mathrm{s}$, respectively. The amount of available averaged measurement points was 23 on the 16.01., 17.01. and 19.01. and 131 points on the 22.01. Table 2 gives an overview of some characteristic parameters for each observation date.

\subsection{TS-X vs LiDAR in free stream conditions}

The top row in Figure 7 illustrates the absolute horizontal wind speed in $10 \mathrm{~m}$ height on the polar standard grid obtained from LiDAR and TS-X on the 22.01. The mean LiDAR wind speed $(6.4 \mathrm{~m} / \mathrm{s})$ is approximately $1.1 \mathrm{~m} / \mathrm{s}$ higher than the one derived from SAR $(5.3 \mathrm{~m} / \mathrm{s})$. It is assumed that the deviation results from inaccuracies in the extrapolation of LiDAR values to $10 \mathrm{~m}$ height caused by non-neutral stratificated atmosphere. The standard deviations of LiDAR and TS-X compare well with $0.39 \mathrm{~m} / \mathrm{s}$ and $0.36 \mathrm{~m} / \mathrm{s}$ respectively. Remarkably, aside the mean speed difference, most of the structures in the flow can be found to be very similar in both plots. Note that the TS-X wind field has a slightly smoother appearance than the LiDAR wind field, which is explained by the lower spatial resolution of the original data being interpolated to the standard grid. These promising results benefit from the low time difference between SAR and LiDAR observations in the regarded case of less than $30 \mathrm{~s}$. An investigation on the beamwise correlation and deviation between the wind fields in dependency to the time delay is carried out for the considered case (cf. Subplot 7.4 in Figure 7). Although the tendency is small, it is noticeable that mean differences (blue) are smallest and the correlation (green) tends to be highest around the TS-X acquisition time (i.e. zero

\begin{tabular}{|c|c|c|c|c|}
\hline Date & 16.01. & 17.01. & 19.01. & 22.01. \\
\hline $\begin{array}{c}\text { Wind Direction } \\
\text { COSMO-DE } \\
\text { (coming from) }\end{array}$ & $174.3^{\circ}$ & $174.5^{\circ}$ & 125.3 & 134.8 \\
\hline $\begin{array}{c}\text { Mean wind speed } \\
\text { COSMO-DE }\end{array}$ & $10.3 \mathrm{~m} / \mathrm{s}$ & $10.8 \mathrm{~m} / \mathrm{s}$ & $12.9 \mathrm{~m} / \mathrm{s}$ & $8.2 \mathrm{~m} / \mathrm{s}$ \\
\hline $\begin{array}{c}\text { Mean Wind Speed } \\
\text { LiDAR }\end{array}$ & $8.8 \mathrm{~m} / \mathrm{s}$ & $10.0 \mathrm{~m} / \mathrm{s}$ & $12.5 \mathrm{~m} / \mathrm{s}$ & $6.4 \mathrm{~m} / \mathrm{s}$ \\
\hline $\begin{array}{c}\text { Mean Wind Speed } \\
\text { TSX }\end{array}$ & $8.4 \mathrm{~m} / \mathrm{s}$ & $6.1 \mathrm{~m} / \mathrm{s}$ & $12.2 \mathrm{~m} / \mathrm{s}$ & $5.2 \mathrm{~m} / \mathrm{s}$ \\
\hline
\end{tabular}

Table 2. Overview of characteristic wind parameters at $10 \mathrm{~m}$ height for model and observations 


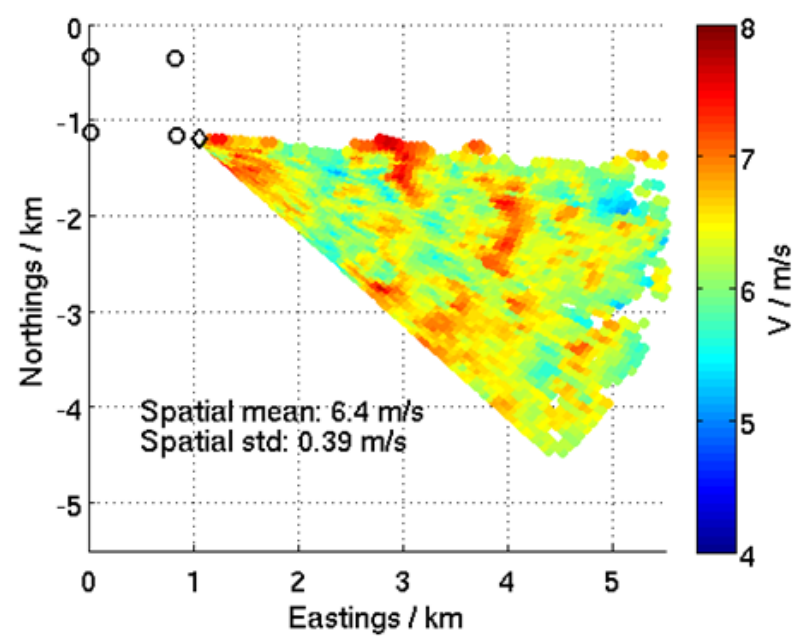

Subplot $(7,1)$. LiDAR-LoS measurements are transformed back to be the absolute wind speed in the ambient wind direction taken from the meso scale weather model COSMO-DE and extrapolated to $10 \mathrm{~m}$ height using a logarithmic wind profile

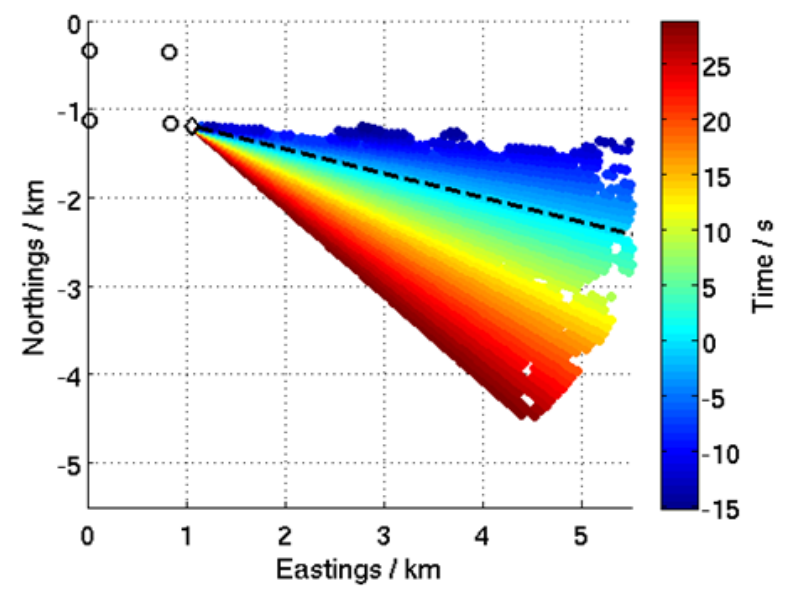

Subplot $(7,3)$. Time difference of LiDAR beams with respect to SAR acquisition time. The time difference between LiDAR and radar measurement was $\leq 30 \mathrm{~s}$. Note that each directional beam has a constant time difference

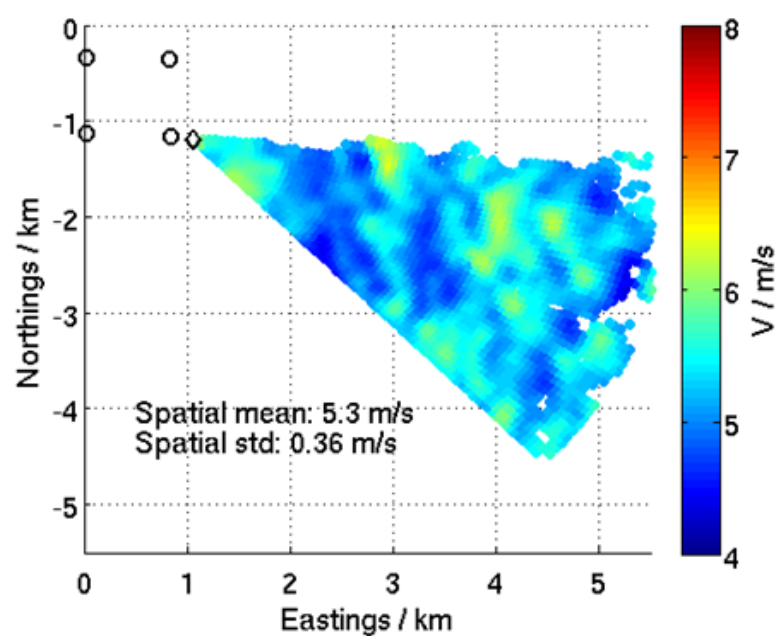

Subplot $(7,2)$. TerraSAR-X wind speeds. The wind direction passed to the algorithm XMOD2 is also taken from the meso scale weather model COSMO-DE

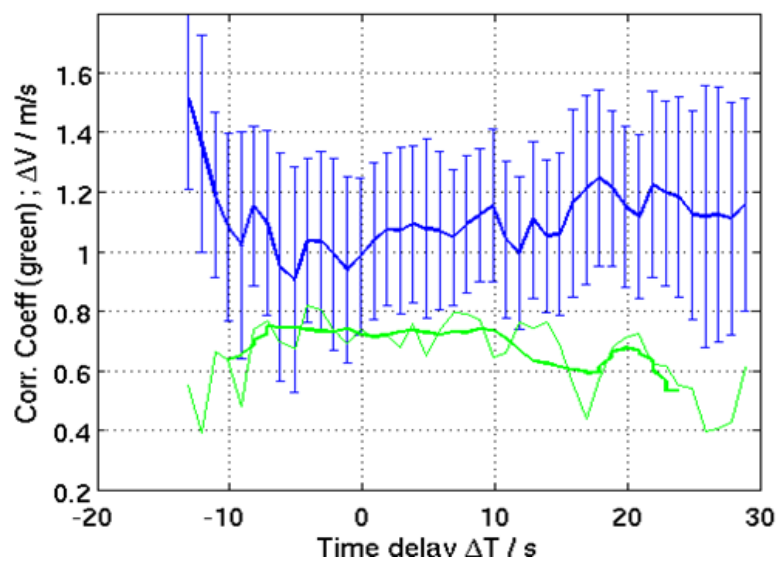

Subplot $(7,4)$. Mean wind speed difference and standard deviation for the separate directional beams with the given time delay (blue). One-dimensional correlation coefficient for each beam (green) with a $5 \mathrm{~s}$ sliding average (bold, green)

Figure 7. Absolute wind fields in $10 \mathrm{~m}$ height upstream of alpha ventus with south easterly wind conditions on the standard grid measured on the 22.01.2014.

seconds time delay).

In Figure 8, the comparison for the LiDAR system stationed on the substation in alpha ventus and TS-X is summarized for all measurement dates. The results show a good agreement of less than $-0.5 \mathrm{~m} / \mathrm{s}$ mean difference between TS-X and LiDAR on January 16 and 19 . On January 22 the difference is still small with approximately $-1.1 \mathrm{~m} / \mathrm{s}$ inside the uncertainty margin of the XMOD-2 accuracy. The mean difference between TS-X and LiDAR is negative in all cases, meaning that for the dates presented in this paper, TS-X systematically measures lower averaged wind speeds in all cases. On January 17, the mean difference reaches values of almost $-4 \mathrm{~m} / \mathrm{s}$. This high mismatch found on the 17.01 is unusual and to some extend puzzling, particularly because LiDAR and COSMO-DE show a good agreement on the given date. The low level of TS-X derived wind seems to originate from an unusually low radar backscatter of $-19 \mathrm{~dB}$, which results in a lower derived wind speed. The scene was acquired at an incidence angle of roughly $49^{\circ}$, which is outside the confirmed confidence margin of the
XMOD2 algorithm. Although high incidence angle wind fields are being investigated, hitherto validation of XMOD2 in this range is not extensive and thus we cannot exclude that the accuracy of wind speed results might suffer. The order of the uncertainty is unfortunately not known. However, on January 19 , the incidence angle was even slightly higher and the results compare well with both COSMO-DE and LiDAR observations. This is a hint that the mismatch cannot be contributed to high incidence angles, only. The low NRCS suggests a very low sea surface roughness despite considerably high wind speeds at LiDAR observation heights. The reasons for the low backscatter level might be related to the ambient meteorological and atmospheric conditions, but needs to be further investigated in detail. However, other image features indicate an unusual situation on January 17: While wind blows from southerly directions, the high resolution spotlight image does not contain any windsea waves in the wind direction, but only exhibits wave structures roughly in east-west direction. Yet, with a wind fetch of several kilometres, the absence of windsea from the 


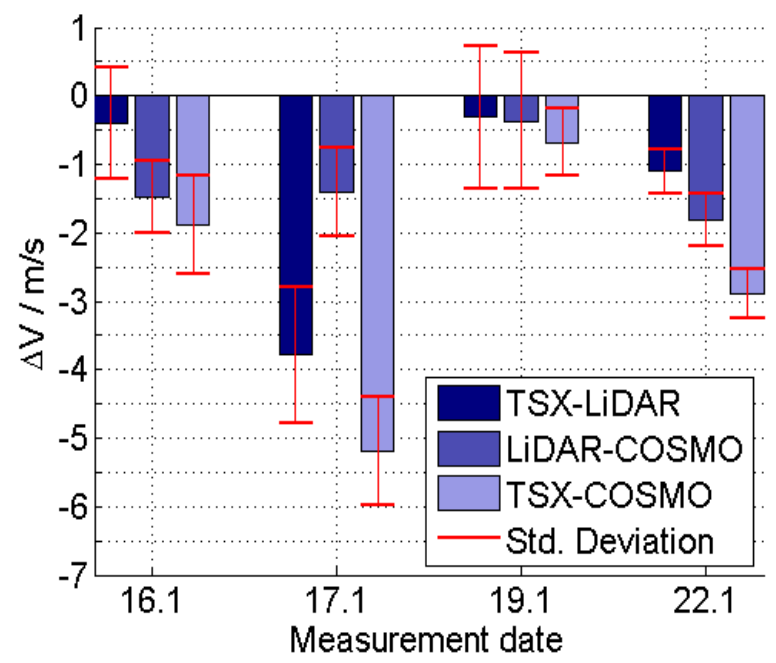

Figure 8. Comparison of absolute wind speeds obtained by TerraSAR-X, LiDAR and COSMO-DE. Mean difference anc standard deviation are plotted

south is unusual. Moreover, radar backscatter values in the turbine wakes show a periodic increase in the wake with respect to the background NRCS. This observation can also not easily be brought into accordance with previous SAR turbine wake observations, where radar backscatter is generally lower. In conclusion, the January 17 data seems to be exceptional in many aspects and should thus be carefully interpreted in the comparison between LiDAR and SAR.

The comparison of the mean LiDAR wind speed to the mesoscale weather model COSMO-DE reveals systematically negative values in all four cases, meaning higher wind speeds obtained by COSMO-DE with respect to LiDAR and SAR. Differences are about $1.5 \mathrm{~m} / \mathrm{s}$ to $1.8 \mathrm{~m} / \mathrm{s}$, on the 19.01 . the difference is lower than $0.5 \mathrm{~m} / \mathrm{s}$. Possible reasons for the observed deviations can be related to the time lag between TS-X and COSMO-DE of up to 23 minutes, the RMSE of COSMO$\mathrm{DE}$ and the applied neutral logarithmic wind profile.

\subsection{Wake}

In Figure 9 the comparison of wake tracks observed with LiDAR and TS-X is presented. The wake is not sharply defined in the TS-X data with a wind speed reduction of $25 \%$ in a wide region downstream of the wind turbine located at $0 \mathrm{D}$. The wake pattern observed by TS-X shows strong meandering behaviour

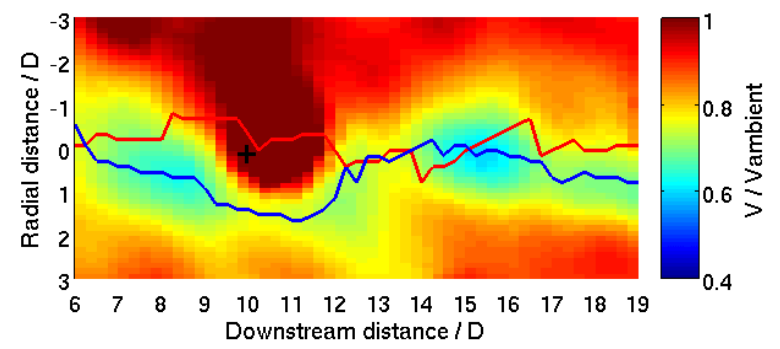

Figure 9. Normalized horizontal wind speed downstream of wind turbine located at $0 \mathrm{D}$ obtained from TS-X measurements (color coded). The centre of the wake track is marked for the lidar measurement (red line) and the corresponding wake track from the TS-X measurement (blue line). The position of a second turbine is marked $(+)$. Both axes are normalized with the rotor diameter $D$ of the wake generating turbine. especially at the region in $10 \mathrm{D}$, where the wake is influenced by the inflow of another turbine. Wake tracks from LiDAR and TS-X do show a maximum deviation of $2 \mathrm{D}$ but are roughly located in the same area. Interestingly a maximum wake deficit with a wind speed reduction of up to $40 \%$ occurs at the regions of $8 \mathrm{D}$ and $16 \mathrm{D}$, which support the idea that the wake fully touches ground after a certain distance downstream of the turbine. However this effect is difficult to evaluate in the dataset here, since the wake is strongly influenced by the inflow and wake of neighboured turbines.

With the resolution of $60 \mathrm{~m}$ in the horizontal plane TS-X resolves just about two data points per rotor diameter $D$ at the regarded turbines $\left(D_{A}=116 \mathrm{~m}\right.$ and $\left.D_{S}=126 \mathrm{~m}\right)$. However, TS$\mathrm{X}$ can yield wind fields of higher spatial resolution, but with drawbacks in the wind speed accuracy. So the localization of the wake tracks is limited by the spatial resolution of the chosen dataset. Another reason for the differences is the time lag between LiDAR measurement and TS-X image of up to $60 \mathrm{~s}$. Considering the fact that the turbulent turbine wake is a dynamic and morphologically heterogenic feature, the comparison of measurements at different times and on different observation heights is cumbersome. Despite these difficulties arising from differences in the location, the duration and time difference and especially the fundamental differences in the measurement principle discussed in section 2.5, the results obtained are quite promising.

\section{CONCLUSIONS}

In this paper we present for the first time a comparison of twodimensional collocated wind fields derived from spaceborne SAR and earth-bound LiDAR. In free stream conditions, a comparison of wind speeds between LiDAR and SAR is performed under the assumption of a neutral logarithmic vertical profile. We observe good agreement in mean wind speed of both systems with a difference of less than $1.1 \mathrm{~m} / \mathrm{s}$ in three of the regarded four cases. While this offset lies within the given accuracy of the SAR wind field derivation, it is probably partly related to the assumption of the neutral stratification of the atmosphere applied in the height extrapolation. With information about the real wind profile obtained from meteorological measurements or from a LiDAR profiler another model could be used to transform the LiDAR wind speed to the level of the TS-X wind field in future campaigns. Despite a minor offset, nearly all free stream wind field variations coincide in LiDAR and SAR data on January 22. This is reflected in the high correlation of the two datasets. A subdivision of the LiDAR data regarding the sample time suggests that the time lag to the SAR snapshot is one source of reduced correlation (c.f. Figure 7). However, the high degree of similarity of most visible flow structures in both methods is remarkable and supports the idea, that in absence of contaminations by other oceanographic phenomena, SAR images can reveal sea surface wind fields and their variations with high resolution and accuracy. However the combination with independent earth-bound remote sensing methods like LiDAR makes it possible to identify disputable cases like January 17. This particular dataset leaves open questions in many respects and will be subject of future investigation.

The comparison of wake tracks revealed low spatial agreement, but looked similar in principle. Due to the higher spatial resolution, LiDAR measurements are better suited to resolve single turbine wakes. Nonetheless, LiDAR observations can be supported by SAR derived wind fields. On the other hand SAR images with the capability to cover large areas are furthermore suited to study the wake of whole offshore wind farms on a bigger scale. Future measurement campaigns will be used to 
compare the wake of a whole offshore wind farm measured with LiDARs to the ones calculated from TS-X data.

\section{ACKNOWLEDGEMENTS}

The lidar measurements in the offshore wind farm alpha ventus were performed in the frame of the research project GW Wakes, funded by the German Federal Ministry for Economic Affairs and Energy (BMWi) based on a decision of the Parliament of the Federal Republic of Germany (grant number 0325397A).

We thank Deutscher Wetterdienst (DWD) for providing the analysis data.

\section{REFERENCES}

Baldauf, M., J. Förstner, S. Klink, T. Reinhardt, C. Schraff, A. Seifert, K. Stephan, and Deutscher Wetterdienst. 2011. "Kurze Beschreibung Des Lokal-Modells Kürzestfrist COSMO-DE (LMK) Und Seiner Datenbanken Auf Dem Datenserver Des DWD.” Deutscher Wetterdienst, Geschäftsbereich Forschung Und Entwicklung, Offenbach, Germany.

Breit, H., T. Fritz, U. Balss, M. Lachaise, A. Niedermeier, and M. Vonavka. 2010. "TerraSAR-X SAR Processing and Products.” IEEE Transactions on Geoscience and Remote Sensing 48 (2): 727-40. doi: 10.1109/TGRS.2009.2035497.

Emeis, Stefan. 2012. Wind Energy Meteorology: Atmospheric Physics for Wind Power Generation. Springer Science \& Business Media.

Frehlich, Rod. 2001. "Estimation of Velocity Error for Doppler Lidar Measurements" Journal of Atmospheric and Oceanic Technology, Vol 18, 1628-1639. doi:10.1175/15200426(2001)018<1628:EOVEFD>2.0.CO;2

Hersbach, H., A. Stoffelen, and S. de Haan. 2007a. “An Improved C-Band Scatterometer Ocean Geophysical Model Function: CMOD5.” Journal of Geophysical Research: Oceans 112 (C3): n/a - n/a. doi:10.1029/2006JC003743.

Horstmann, Jochen, Wolfgang Koch, and Susanne Lehner. 2004. "Ocean Wind Fields Retrieved from the Advanced Synthetic Aperture Radar Aboard ENVISAT.” Ocean Dynamics 54 (6): 570-76.

Lehner, S., J. Horstmann, W. Koch, and W. Rosenthal. 1998. "Mesoscale Wind Measurements Using Recalibrated ERS SAR Images.” Journal of Geophysical Research: Oceans, 103 (C4): 7847-56. doi:10.1029/97JC02726.

Li, Xiao-Ming, and S. Lehner. 2013. "Observation of TerraSAR-X for Studies on Offshore Wind Turbine Wake in Near and Far Fields." IEEE Journal of Selected Topics in Applied Earth Observations and Remote Sensing 6 (3): 175768. doi:10.1109/JSTARS.2013.2263577.

Li, Xiao-Ming, and Susanne Lehner. 2014. “Algorithm for Sea Surface Wind Retrieval From TerraSAR-X and TanDEM-X Data." IEEE Transactions on Geoscience and Remote Sensing 52 (5): 2928-39. doi:10.1109/TGRS.2013.2267780.

Peña, Alfredo, Charlotte Bay Hasager, Sven-Erik Gryning, Michael Courtney, Ioannis Antoniou, and Torben Mikkelsen. 2009. "Offshore Wind Profiling Using Light Detection and Ranging Measurements.” Wind Energy 12 (2): 105-24. doi:10.1002/we.283.
Sempreviva, A. M., R. J. Barthelmie, and S. C. Pryor. 2008. "Review of Methodologies for Offshore Wind Resource Assessment in European Seas.” Surveys in Geophysics 29 (6): 471-97. doi:10.1007/s10712-008-9050-2.

Stawiarski, Christina. Träumner, Katja. Knigge, Christoph. Calhoun, Ronald. 2013. "Scopes and Challenges of DualDoppler Lidar Wind Measurements - An Error Analysis”, Journal of Atmospheric and Oceanic Technology, Vol 30, 2044-2062. doi: 10.1175/JTECH-D-12-00244.1

Stoffelen, A., Anderson, D., 1997. "Scatterometer Data Interpretation: Estimation and Validation of the Transfer Function CMOD4.” Journal of Geophysical Research 102 (C3): 5767-80.

Trabucchi, D., Beck, H., Schneemann, J., Trujillo, J. J., Ungurán; Róbert; Stephan, V. \& Kühn, M., “Offshore wind farm flow characteristics measured by a long range multi lidar system”, EWEA Offshore 2013, 2013

Werner, Christian. 2005. "Doppler Wind Lidar." In Lidar, edited by Claus Weitkamp, 102:325-54. Springer Series in Optical Sciences. Springer Berlin / Heidelberg. 